\title{
Communication Management and Control in Distance Learning Scenarios
}

\author{
Freimut Bodendorf and Manfred Schertler \\ University of Erlangen-Nuremberg, Nuremberg, Germany
}

\section{bodendorf@wiso.uni-erlangen.de schertler@wiso.uni-erlangen.de}

\begin{abstract}
An approach to improve communication processes in e-learning is introduced. Based on a separation of a content-related and a communicational part of e-learning environments a system to support both students and tutors in their communication endeavors is presented. The communication platform consists of administration, configuration, moderation, and certainly communication tools which are described in detail. Finally the example of a telecooperative case study is given to illustrate the platform's potential in specific e-teaching/e-learning scenarios.
\end{abstract}

Keywords: Distance Education, Communication Control, Collaborative Learning, Tutoring Support

\section{Introduction}

Communication plays a major role in successful e-learning scenarios. Most of the e-learning or learning management software environments available on the market offer at least some kind of communication support. But the focus of e-education still lies on producing content and distributing course material via the Internet. In spite of the fact that knowledge is growing faster and faster and the need for e-learning that is up-to-date is obvious, just providing well structured and preprocessed content is not sufficient to fulfill the goals of modern education. Only discussing, applying and working with the acquired new knowledge builds up the competencies that students need to face the challenges of their professional and private life (Salmon, 2000).

An approach to place emphasis on communication in contrast to just providing content is to separate content-related and communicational aspects of learning environments. This approach is part of the e-learning research activities at the Department of Information Systems at the University of Erlangen-Nuremberg (Schertler \& Bodendorf, 2002). Besides the need for content students demand intellectual exchange, individual advice, and personal guidance. Based on elementary communication patterns and an effective and efficient technical infrastructure a set of reference communication processes has been created to be carried out depending on the chosen teaching method (for example lectures, exercises, tutorials, case studies, business games, role plays and so forth).

Material published as part of this journal, either on-line or in print, is copyrighted by Informing Science. Permission to make digital or paper copy of part or all of these works for personal or classroom use is granted without fee provided that the copies are not made or distributed for profit or commercial advantage AND that copies 1) bear this notice in full and 2) give the full citation on the first page. It is permissible to abstract these works so long as credit is given. To copy in all other cases or to republish or to post on a server or to redistribute to lists requires specific permission from the publisher at Publisher@InformingScience.org
A support system for so called scheduled learning in e-learning scenarios is presented. Scheduled learning is a paradigm that is mainly based on a tight time structure (schedule) of an elearning process. The term scheduled refers to a precise and detailed placement of learning and teaching acts 
within one educational scenario. The schedule has the following objectives:

- Each participant, either student or tutor, is able to determine at any time, which tasks are handled at the moment and which tasks are to be solved in the near future. A possible "lost-in-elearning", similar to "lost-in-hyperspace", can be prevented.

- Organization of group learning processes is simplified. The schedule eases the coordination of sub-processes like assigning tasks to group members, making decisions on group learning strategies and so forth.

- Communication processes between tutors and students as well as among students themselves are carried out in a more systematic way. Communication problems not particularly concerning the contents of an e-learning course are reduced.

- Mapping of supporting tools with teaching or learning acts is facilitated. The tool allocation makes more sense and learners as well as tutors are not overstrained by an excessive supply of communication channels. The development of communication process descriptions and corresponding communication channel support is mainly task of full-time didactical designers in an educational institution. As regular tutors often are seasonal staff they may neither have the expertise nor the time to define communication processes and tool support by themselves.

E-learning scenarios live on a tight over-all organization. Disordered communication processes lower learning success and motivation to go on with a course. A schedule of default actions supports target-oriented processes and learning flows.

\section{Problem}

In a traditional classroom many communication problems do not arise because they dissolve en passant. Tutors and students use verbal, non-verbal and paraverbal techniques to channel their communication acts either on purpose or by incident. In e-learning scenarios conditions are different. Problems emerge from temporal and regional distribution, the broad variety of so-called supportive tools and a lack of guidance during communication (Schertler \& Bodendorf, 2002). The most outstanding problems are:

- Tutors have problems to react to student's behavior in a proper way because they cannot rely on non- or paraverbal behavior (Boudourides, 1995) as they do in conventional courses to control the class.

- If there is only limited or no anonymity students won't take part in an web-based learning experience as desired because they may not want to exhibit their learning results to the whole audience (Bodendorf \& Schertler, 2000).

- E-learning communication scenarios often loose the line of argumentation because of transmission or handling troubles.

- Without appropriate social surroundings a continuous discussion is difficult to maintain at a high level.

- The choice of a fitting communication tool for a specific communication purpose is often underestimated. For example, coaching an entire course with e-mail undermines the huge oneto-one potential of this communication channel (Baltes, 2001; Paulsen, 1999).

- To organize a computer-mediated discussion and to keep it running a remarkable amount of time and motivation is needed in advance of the actual discussion. 
- A huge amount of miscellaneous comments is a direct consequence of lacking guidance which leads to meaninglessness instead of effective discussions.

Below the technical deficiencies of existing e-learning environments in matters of communication support are highlighted from a users' point of view. Four popular e-learning and communication platforms are outlined to depict their shortcomings. The following descriptions come from handson experiences of online tutors at several virtual universities (virtual university of Bavaria: http://www.vhb.org, virtual global university: $h t t p: / / w w w . v g-u . d e)$.

\section{- Blackboard (http://www.blackboard.com)}

In every Blackboard course the communication support tools can be reached via a button on the left-handed navigation bar. In addition, the so-called virtual classroom, a discussion board and group specific pages can be selected using designated buttons. Besides this, an e-mail function is an integrated part of the system as well as a roster.

Default communication channels are only available when a tutor places an announcement on the top page of a course and links the appropriate communication channel in this announcement. Otherwise the students have to pick the tool they want or need to use by themselves. The tools offer standard functionality without special moderating support like bad-word filters or floor control for the tutors. E-mail and discussion boards fulfill asynchronous communication needs, the virtual classroom offers a combined synchronous chat and whiteboard channel with some kind of personal view support (so-called user info).

The communication tools offered by Blackboard cover the most frequently used channels in elearning communication. Nevertheless each single channel supports structured e-conversation only to a small extent. Suggestions of reasonable combinations of communication matter and communication channel are only given implicitly in case of synchronous communication, when a text chat is combined with a graphical shared application whiteboard. At least the possibility to facilitate the organization of communication processes is accomplished by pointing to the recommended channels within the announcement section of a course.

\section{- WebCT (http://www.webct.com)}

Similar to Blackboard WebCT lists all communication channels in its navigation area. Discussion forums, e-mail, and chats are the ways students and tutors can communication via this software. Within the channels the common basic functions are implemented but no special features to support moderated discussions or organizing communication processes in a more sophisticated way than just assigning messages to a comprising topic are integrated.

The evaluation of WebCT's communication environment reveals a rather weak support level. The channels are more or less only provided; the success of a communication process strongly depends on the capabilities of the tutor or author of a course.

\section{- Lotus Learning Space (http://www.lotus.com/learningspace)}

Lotus Learning Space offers e-mail and chat functionality but furthermore a sophisticated screen sharing and video conferencing option. Unfortunately the synchronous tool is a little bit overloaded so that a participant on novice level (regarding his computer-mediated communication skills) might be swamped with the plurality of possibilities. A positive aspect of this environment is the so-called course list which itemizes all course contents and in addition the students actions over a specific period of time. This concept resembles the idea of a scheduled learning process.

- Netmeeting (http://www.microsoft.com/windows/netmeeting)

Netmeeting represents a pure communication software and offers a broad range of communication channels reaching from simple text chat messages over screen and application sharing possi- 
bilities to full video conferencing support. But despite this potential Netmeeting is not designed for educational purposes in the first place. The spectrum of communication tools is not easy to overlook, there is no connection between single communication channels, no process support, and no suggestion of an adequate communication channel selection for a specific communication purpose.

To sum up, the scheduling aspect is not part of most of the communication software solutions yet. Tutors seem to have very few possibilities to influence communication processes or to take moderation actions. These actions are not used and/or supported sufficiently.

\section{Communication Channels}

In the following section the main communication channels of the presented system are described,

\begin{tabular}{|c|c|c|c|}
\hline $\begin{array}{l}\text { Communica- } \\
\text { tion channel }\end{array}$ & Features & Moderation functions & User functions \\
\hline E-mail & $\begin{array}{ll}\text { - } & \text { usually one-to-one } \\
\text { - } & \text { asynchronous } \\
\text { - } & \text { mainly private } \\
& \text { storage medium } \\
\text { - } & \text { mandatory } \\
& \text { separation of structure } \\
\text { and content }\end{array}$ & $\begin{array}{ll} & \text { automatic mail in case } \\
& \text { of forum activities (new } \\
\text { - } & \text { postings, statistics) } \\
\text { - } & \text { ready-made mail forms } \\
\text { - } & \text { cry filter } \\
\text { - } & \text { contribution size control } \\
\text { - } & \text { smiley support } \\
\end{array}$ & $\begin{array}{l}\text { student: new e-mail, } \\
\text { delete e-mail, read e- } \\
\text { mail, answer e-mail, } \\
\text { organize inbox } \\
\text { tutor: broadcast e- } \\
\text { mail }\end{array}$ \\
\hline Forum & $\begin{array}{ll}\text { - } & \text { usually one-to-many } \\
\text { - } & \text { asynchronous } \\
\text { - } & \text { mainly public } \\
\text { - } & \text { storage medium } \\
& \text { mandatory } \\
\text { - } & \text { separation of structure } \\
& \text { and content } \\
\text { - } & \text { tree structure ensures } \\
& \text { line of argumentation }\end{array}$ & $\begin{array}{ll}\text { - } & \text { bad-word filter } \\
\text { - } & \text { cry filter } \\
\text { - } & \text { contribution size control } \\
\text { - } & \text { e-mail notification } \\
\text { - } & \text { smiley support } \\
\text { - } & \text { contribution ranking } \\
\text { - } & \text { statistics with auto- } \\
& \text { mailer functionality } \\
\end{array}$ & $\begin{array}{l}\text { student: open forum, } \\
\text { make contribution, } \\
\text { delete own contribu- } \\
\text { tion, organize forum } \\
\text { structure } \\
\text { tutor: new forum, } \\
\text { delete forum, show } \\
\text { statistics, send statis- } \\
\text { tics }\end{array}$ \\
\hline Chat & $\begin{array}{ll}\text { - } & \text { usually many-to-many } \\
\text { - } & \text { synchronous } \\
\text { - } & \text { private or public } \\
& \text { storage medium op- } \\
& \text { tional }\end{array}$ & $\begin{array}{ll}- & \text { ready-made notifica- } \\
& \text { tions } \\
- & \text { floor control } \\
\text { - } & \text { bad-word filter } \\
\text { - } & \text { cry filter } \\
& \text { contribution length con- } \\
\text { - } & \text { trol } \\
\text { smiley support }\end{array}$ & $\begin{array}{l}\text { - } \\
\text { student: access mes- } \\
\text { sage history, post } \\
\text { message, apply for } \\
\text { contribution right, } \\
\text { unblock contribution } \\
\text { right, print chat log } \\
\text { tutor: grant contribu- } \\
\text { tion right, withdraw } \\
\text { contribution right }\end{array}$ \\
\hline Whiteboard & $\begin{array}{ll}\text { - } & \text { usually many-to-many } \\
\text { - } & \text { synchronous } \\
\text { - } & \text { private or public } \\
\text { - } & \text { storage medium op- } \\
& \text { tional }\end{array}$ & $\begin{array}{ll}- & \text { document import } \\
\text { - } & \text { floor control } \\
\text { - } & \text { bad-word filter } \\
\text { - } & \text { cry filter }\end{array}$ & $\begin{array}{l}\text { student: new docu- } \\
\text { ment, import docu- } \\
\text { ment, save document, } \\
\text { draw, print docu- } \\
\text { ment, apply for con- } \\
\text { tribution right, return } \\
\text { contribution right } \\
\text { tutor: grant contribu- } \\
\text { tion right, withdraw } \\
\text { contribution right }\end{array}$ \\
\hline
\end{tabular}


their special features are pinpointed and moderating functions are shown.

Table 1 shows the features, moderation and user functions of four communication channels.

The channels of the communication platform are broken down into synchronous and asynchronous communication formats. During synchronous communication (chat, whiteboard) two or more participants communicate at the same time within the system. During asynchronous communication (e-mail, discussion forum) there is no need for both communication partners to be online at the same time, because messages are stored in the system over a longer period of time (Berge \& Collins, 1995).

To save the contents of synchronous discussions for later re-use the system provides archive and print functionalities in the chat and whiteboard channel. A message history supports participants who joined a chat session later and missed parts of the conversation. Floor control ensures the line of argumentation and the reference between contributions.

To enhance the functionality of the communication channels additional moderation functions have been implemented. A bad-word filter compares each message with a list of forbidden expressions. In case of a bad-word violation the system does not send the message. The cry filter works in a similar way. Words written in capital letters are blocked. Excessive length of contributions is inhibited by a maximum number of characters of a single message. In addition, the time between two contributions can be set to a minimum, so that each participant has to wait a few moments before he can post again. This prevents users from sending only fragments of messages. These users often try to accroach a conversation by unnecessarily increasing their number of contributions and segregating other participants.

\section{Designing Communication Processes}

A critical success factor for every learning process is the appropriate correlation between communication channel and educational purpose. A theoretical background to fulfill this task can be found in the media synchronicity theory (Dennis \& Valacich, 1999) and the task media fit model (Buder, 2000). Both theories help to eliminate the inadequate directive and guidance options respectively the missing configuration possibilities of existing systems.

The support system presented in this paper concentrates on managing a structured support of learning processes as well as on a purposeful mapping of communication tools. One major goal is to define basic communication processes and to use these definitions to configure the communication channels The tutor or moderator needs to set parameters for each channel according to the current task in order to avoid too restricted or too open communication processes.

In a communication process participants attend partially or completely moderated courses. Courses are based on certain teaching methods and consist of recurring learning phases. As courses can be handled like communication processes these learning phases can be denoted as communication patterns. Each pattern within one process supports a specific task which is characterized by a typical communication situation. To facilitate a certain task each pattern is linked to the communication channels that ensure a worthy exchange and acquisition of knowledge.

The tutor is able to define new processes or to use predefined templates for a specific course configuration. Each template is assigned to a category (for example general presentation, case studies, management games, project work, and so forth) to be easily detected by an inexperienced tutor.

The following steps are proposed to define a communication process: 
- Choose communication patterns und define pattern sequence: The tutor chooses the communication patterns he likes to design the communication process with (for example warm-uppattern, presentation pattern, discussion pattern, and so forth). The sequential order of the patterns is similar to a workflow definition within a workflow management system.

- Define attributes of communication patterns: Each communication pattern has specific characteristics to fulfill its tasks in the over-all communication process. The tutor determines the trigger type, which enables tutors and/or students to switch to the next phase and communication pattern in the process. Possible triggers are tutor control, student control or time control. Additionally the tutor can compose help texts and work instructions for each pattern. Based on the theoretical background the system also suggests reasonable communication channels for the current pattern.

- Assign communication channels: As mentioned before each pattern comprises suggestions of communication channels that suit the tasks of a pattern best. But the actual decision of assigning a communication channel to a communication pattern is incumbent upon the tutor.

- Define attributes of communication channels: The selected communication channels are provided with moderation functions and an additional help text from the tutor.

After a communication process is completely defined it is given a convenient name and saved as a template to use and re-use in several courses.

\section{User Interface}

Figure 1 shows a user interface screenshot of the communication platform with two active channels, chat and whiteboard.

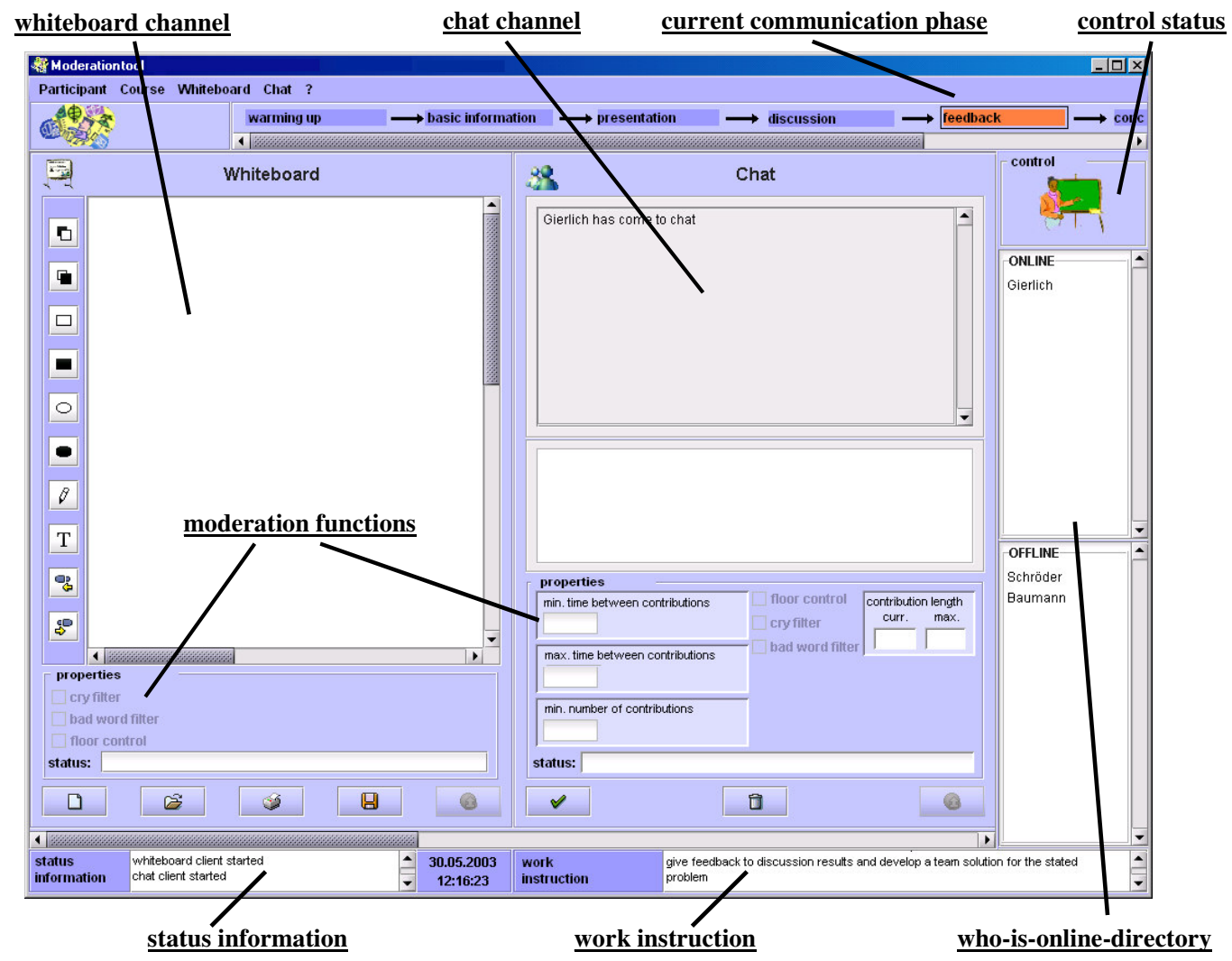

Figure 1: User interface of the communication platform 
A menu bar is located on top of the screen. Menu items include login and logoff functions, course and participant information (personal views) and help options referring to the communication channels of the current communication pattern. Beneath the menu bar the communication process of the current course is displayed, the active pattern is highlighted. This overview helps students to realize the status quo of the over-all process.

A major part of the user interface is reserved for the automatically displayed communication channels. Figure 1 for example shows a whiteboard combined with a text chat.

In the lower part of the screen a status information area and the work instructions of the current communication pattern are provided. On the right side of the communication channels the control status (learner control, tutor control, time control) for the current pattern is displayed. This area fulfills either a mere information task (in case of tutor or time control) or offers the student a pattern control button to indicate a desired pattern switch. Not until all students agree on switching to the next pattern the platform carries out this operation.

In addition the user interface comprises a who-is-online-directory that shows all participants of a course and their online status. A double click on a name reveals his or her personal information.

\section{Example: Supporting Communication Processes in a Telecooperative Case Study}

The example of a telecooperative case study (Bodendorf \& Schertler, 2000; Schertler \& Bodendorf, 2002) from a technical point of view illustrates the effectiveness and usability of the presented communication platform. Case studies are well-known and successful methods in didactical settings which focus on different competencies like gathering and analyzing information, developing solutions, and making decisions. Communication during a case study consists of one-tomany communication between the presenter of the case and the students, many-to-many communication during discussions, and asynchronous communication scenarios. During case studies interaction between tutors and students as well as interaction between the students themselves is probably the most important part of the whole learning process. Only a well balanced interaction can reach the high expectations resulting from the use of case studies in higher education arrangements (McIsaac \& Gunawardena, 1996).

A typical telecooperative case study consists of seven phases (Bodendorf \& Schertler, 2000):

- Warming up: An overview of the case and the influencing factors is given.

- Case presentation: The case, its major facts and circumstances, and possible questions to be answered by the students are presented by a tutor or a company's representative.

- Background information: Students analyze the case information and gather additional information from various sources, for example the Internet.

- Individual solutions: Each student develops a solution approach for the case and answers to the given questions.

- Discussion, team solutions: All students are divided into teams. Each team combines the individual solutions of its members.

- Group solution: Each team presents its solution elaborated on the basis of the team members' individual solutions.

- Final discussion, feedback: All team solutions are discussed among the students and together with the tutor and maybe with the company's representative. 
During all phases and in all communication channels bad-word and cry filters are applied. Each phase is described by a help text, and a work instruction sums up the necessary actions all participants of a phase have to fulfill to successfully finish the phase and step to the next one.

The first phase (warming up) is supported by a discussion forum and the phase control is timebased. A tutor, maybe together with a company's representative, posts first information into a forum thread. All students should read and try to comment on these postings to become involved with the case environment. The presentation of the case is tutor controlled and supported by the same forum thread as well as a chat and whiteboard. The discussion forum is used by the presenter of the case to supply the students with the case description. The whiteboard assists the presenter to illustrate the case problems with ready-made or spontaneous sketches or drawings. In case of immediate information needs the students use the chat to broach the subject again or to comment on the case. To enhance synchronous discussion no restrictive moderation functions like floor-control or length limitation of contributions are applied during this phase. In the third phase (background information) asynchronous communication channels are dominant. A new forum thread is used to exchange information among all students, e-mail is used for special requests to the tutor. This phase as well as the following one is time controlled, the tutor defines a date when the actions of these phase have to be finished. In phase four (individual solutions) communication is limited to occasional e-mail contacts as the main focus of the phase is individual work. An own solution for the case is developed by each student. In contrast, the next phase (team solution) is determined by vivid discussions among the team members who are in need of creating one team solution out of many individual approaches. The phase is supported by chat and whiteboard and is student controlled in the first place (i.e., only until all group members agree the phase will be finished). But to keep the discussion topic focused a number of moderation functions are applied, for example the size of a contribution is limited (for example 200 characters) to prevent individual students from taking over the discussion or a minimum time delay between contributions of the same person is set (for example 30 seconds or one minute). Phase six (group solution) is very similar to phase two but now the students present their team solutions to the tutor and/or the company's representative using chat, whiteboard, and a forum thread. In the last phase (final discussion) the tutor comments on the solutions, presents real life aspects of the case environment and sums up the whole case study together with the student teams. They work with the same communication channels as in phase six. Both finishing phases are tutor controlled.

\section{Findings}

To analyze communication processes between tutors and students an empirical study has been carried out during the summer term of the year 2003. Major goal of the study was to find basic relationships between communication processes and the usage of communication tools in virtual educational settings.

The study has been conducted among students of online courses offered by the Virtual University of Bavaria (VHB). A total of 19 students (12 female, 7 male) completed the anonymous online questionnaire. Below a selection of findings is presented related to given hypotheses:

- Students use the communication tool they are familiar with: To verify this hypothesis, data related to communication tool usage has been collected using the question: How often have you used the communication channels e-mail, chat, forum? In addition, the students have been asked about their communication preferences previous to the online course. Combining these two datasets reveals that 11 of 13 students with a tool preference used that tool already. Thus, it is important to detect students' preferences while configuring the communication platform. 
- Experienced users contribute more than inexperienced students: Here, the assumption is that students with higher computer skills overcome inhibitions regarding new communication tools easier than computer newcomers. The students specified their computer skills (question: Please rate your computer skills on a scale from 1-lowest to 5-highest) and the total number of individual contributions within the communication platform. These datasets show that all students with good or very good computer skills made more than 20 contributions whereas 4 of 6 students with medium or low computer skills hadn't posted a contribution at all. Inexperienced users need special support provided by tutors and/or maybe a communication platform.

- Anonymity doesn't facilitate online communication: The communication platform requires registration. Therefore the students have been asked: Do you care about being anonymous in the Internet in general and within the communication platform in particular? 15 of 19 students state that anonymity in the Internet is important. 4 students think being anonymous is of only medium importance. But just 3 students guess that anonymity would increase the number of contributions within the communication platform. The rest of them doesn't mind being known while communicating in an online course.

- A platform with scheduled processes eases communication: To find out whether this hypothesis is right or wrong the students have been asked: Would you appreciate it if default communication tools are provided by a communication platform automatically in certain learning situations? Only 6 of 19 students dislike the vision of always taking part in scheduled communication processes. All of them have good or very good computer skills. This result confirms the findings related to the second hypothesis. Referring to this small empirical study first of all inexperienced users profit from a communication platform with scheduled communication processes.

As an additional result the students suggested that introducing and explaining the whole functionality of a communication tool would enhance both acceptance and intensity of utilization of that tool. For example it is possible to start chat sessions with a short kick-off phase where each student and tutor has to introduce him- or herself using the chat functions. This kick-off idea could be used for other tools as well, for example within a discussion forum. After the introduction phase each participant knows how to use a tool correctly and is motivated to contribute frequently to the rest of the course.

\section{Conclusion}

A way to improve communication quality in e-learning scenarios is presented. Based on the analysis of obvious inconveniences of communication processes and communication tools a tight, scheduled support structure is applied to the communication processes of students and tutors. An integrated communication platform offers appropriate communication channels according to the actual communication needs of a student or tutor.

Further research work focuses on the development of an extensive template library containing reference communication processes for various didactical methods and teaching arrangements. From a technical point of view the platform, which at the moment is implemented as a Java based application, needs to be transferred to a web-based solution to ease access and handling for the participating students. As a next step the communication platform will be integrated in an elearning portal where communication, content, assessments, exercises, and so forth can be organized, administered, and controlled as a whole. 


\section{References}

Baltes, B. (2001). Online-lernen, Schwangau: Huber.

Berge, Z. L., \& Collins, M. P. (1995). Computer-mediated communication and the online classroom. New Jersey: Hampton Press.

Bodendorf, F., \& Schertler, M. (2000). Media based cooperative teaching between university and industry. In Research and innovation in open and distance learning. Book of Essays, 1st EDEN Research Workshop, Prague.

Boudourides, M. A. (1995). Social and psychological effects in computer-mediated communication. Retrieved February 27, 2004 from http://www.math.upatras.gr/ mboudour/articles/csi.html

Buder, J. (2000). Wissensaustausch und wissenserwerb in computerkonferenzen - Der einfluss des metawissen. University of Tübingen.

Dennis, A., \& Valacich, J. (1999). Rethinking media richness: Towards a theory of media synchronicity. In: Proceedings of the 32nd Annual Hawaii International Conference on System Sciences.

McIsaac, M. S., \& Gunawardena, C. N. (1996). Distance education. In: D. H. Jonassen, Handbook of research for educational communications and technology, A project of the Association for Educational Communications and Technology. New York: Macmillan.

Paulsen, M. F. (1999). The online report on pedagogical techniques for computer-mediated communication. Retrieved May 15, 2003 from http://www.nettskolen.com/pub/artikkel.xsql?artid=123

Salmon, G. (2000). E-moderation - The key to teaching and learning online. London: Kogan Page.

Schertler, M., \& Bodendorf, F. (2002) Supporting communication processes in e-learning networks. In Proceedings of ICCE 2002, Auckland, Volume 1.

\section{Biographies}

Freimut Bodendorf is full professor and head of the Department of Information Systems at the University of Erlangen-Nuremberg, Germany.

Manfred Schertler is a member of research staff and Ph.D. student at the Department of Information Systems at the University of Erlangen-Nuremberg. 